\title{
Separation properties for self-conformal sets
}

\author{
by \\ Yuan-Ling Ye (Hong Kong and Guangzhou)
}

\begin{abstract}
For a one-to-one self-conformal contractive system $\left\{w_{j}\right\}_{j=1}^{m}$ on $\mathbb{R}^{d}$ with attractor $K$ and conformality dimension $\alpha$, Peres et al. showed that the open set condition and strong open set condition are both equivalent to $0<\mathcal{H}^{\alpha}(K)<\infty$. We give a simple proof of this result as well as discuss some further properties related to the separation condition.
\end{abstract}

1. Introduction. Let $U_{0} \subset \mathbb{R}^{d}$ be a bounded open set. Let $w_{j}: U_{0} \rightarrow U_{0}$ $(j=1, \ldots, m)$ be contractive maps and suppose there exists a nonempty compact subset $X \subseteq U_{0}$ such that $w_{j}(X) \subseteq X$ for each $1 \leq j \leq m$. Then there exists a compact subset $K \subseteq X$ such that $K=\bigcup_{j=1}^{m} w_{j}(K)$. We say that $\left\{w_{j}\right\}_{j=1}^{m}$ satisfies the open set condition (OSC) if there exists a nonempty bounded open set $U \subseteq U_{0}$ such that

$$
w_{j}(U) \subseteq U \text { and } w_{i}(U) \cap w_{j}(U)=\emptyset \quad \text { for } i \neq j .
$$

Such a $U$ is called a basic open set for $\left\{w_{j}\right\}_{j=1}^{m}$. If moreover $U \cap K \neq \emptyset$, then $\left\{w_{j}\right\}_{j=1}^{m}$ is said to satisfy the strong open set condition (SOSC). In [S], Schief made use of an idea of Bandt [BG] and showed that for similitude, the two conditions are equivalent, and furthermore they are equivalent to $0<\mathcal{H}^{\alpha}(K)<\infty$ where $\alpha$ is the similarity dimension of $K$.

Recently, Peres, Rams, Simon and Solomyak [P] extended Schief's theorem to self-conformal maps. A simple proof was also given by Lau, Rao and the author for the equivalence of the OSC and SOSC [L]. In a private communication, Peres asked if there is a short proof of the equivalence to $0<\mathcal{H}^{\alpha}(K)<\infty$. In this note we answer his question affirmatively. The main idea and some of the proofs are already in $[\mathrm{L}]$ and $[\mathrm{FL}]$; we will modify them to fit our purpose. In [LX] Lau and Xu considered the boundary dimension of self-similar sets. We extend some of their results to self-conformal maps.

2000 Mathematics Subject Classification: Primary 28A78, 54E40; Secondary 54H15.

Key words and phrases: self-conformal set, conformality dimension, OSC, SOSC, basic open set, Hausdorff dimension.

The research was partially supported by a direct grant of CUHK and the NSF of Guangdong. 
For one-to-one contractive self-conformal IFS $\left\{w_{j}\right\}_{j=1}^{m}$, we define the conformality dimension of the IFS to be the (positive) number $\alpha$ such that the Ruelle operator $T_{\alpha}: C(K) \rightarrow C(K)$ defined by

$$
T_{\alpha} f(x)=\sum_{j=1}^{m}\left|w_{j}^{\prime}(x)\right|^{\alpha} f\left(w_{j}(x)\right)
$$

has spectral radius 1 [FL]. We let $\mathcal{H}^{\alpha}$ be the $\alpha$-Hausdorff measure.

We prove Theorem 1.1 below by constructing a basic open set $U$ which satisfies both the SOSC and $\operatorname{dim}_{H}(K \backslash U)<\alpha$. The key to the proof is Lemma 3.4. Furthermore we remark that in the previous considerations of self-conformality, it was additionally assumed that the open set $U$ in the OSC is connected (see e.g. [MU], [P]); we will see that this assumption is redundant (Lemma 2.1 and the remark there). Our basic results are:

THEOREM 1.1. Let $\left\{w_{j}\right\}_{j=1}^{m}$ be a one-to-one self-conformal contractive IFS with $\left\{\left|w_{j}^{\prime}(x)\right|\right\}_{j=1}^{m}$ satisfying (2.1) and the Dini condition. Then the following are equivalent:

(i) $\left\{w_{j}\right\}_{j=1}^{m}$ satisfies the OSC.

(ii) $\left\{w_{j}\right\}_{j=1}^{m}$ satisfies the SOSC.

(iii) $0<\mathcal{H}^{\alpha}(K)<\infty$.

TheOREM 1.2. Let $\left\{w_{j}\right\}_{j=1}^{m}$ be as in Theorem 1.1 and satisfy the OSC. Then there exists a basic open set $U$ such that $\operatorname{dim}_{H}(K \backslash U)<\alpha$.

2. Preliminaries. Let $\left\{w_{j}\right\}_{j=1}^{m}$ be self-conformal on $U_{0}$ (i.e. for each $j$ and each $x \in U_{0}, w_{j}^{\prime}(x)$ is a self-similar matrix and $\left|w_{j}^{\prime}(\cdot)\right|$ is continuous). We assume that there exists a nonempty compact set $X$ such that $X \subseteq U_{0}$, and for each $1 \leq j \leq m, w_{j}(X) \subseteq X, w_{j}$ is one-to-one on $U_{0}$ and $\left|w_{j}^{\prime}(\cdot)\right|$ is Dini continuous on $U_{0}$ with

$$
0<\inf _{x \in U_{0}}\left|w_{j}^{\prime}(x)\right| \leq \sup _{x \in U_{0}}\left|w_{j}^{\prime}(x)\right|<1 \quad \text { for each } 1 \leq j \leq m,
$$

where $\left|w_{j}^{\prime}(x)\right|:=\left|\operatorname{det} w_{j}^{\prime}(x)\right|^{1 / d}$ is the operator norm of the matrix $w_{j}^{\prime}(x)$ on $\mathbb{R}^{d}$. Enlarging $X$ to $X_{0} \subseteq U_{0}$ by taking a $\delta$-neighborhood, we can show easily from the contractiveness of $w_{j}$ 's that there exists $k^{\prime}$ such that

$$
\bigcup_{|J|=k} w_{J}\left(X_{0}\right) \subseteq X_{0} \quad \text { for any } k \geq k^{\prime}
$$

where $J=j_{1} \ldots j_{k}, 1 \leq j_{i} \leq m, w_{J}=w_{j_{1}} \circ \ldots \circ w_{j_{k}}$. Hence we may assume without loss of generality that $\overline{X^{\circ}}=X$ and $B(K, \delta) \subseteq X^{\circ}$ for some $\delta>0$ $(B(K, \delta)$ denotes the open $\delta$-neighborhood of $K)$. 
We set $\mathcal{J}=\left\{J=j_{1} \ldots j_{n}: 1 \leq j_{i} \leq m, n \in \mathbb{N}\right\}$, and for any $J \in \mathcal{J}$ define

$$
K_{J}=w_{J}(K), \quad r_{J}=\inf _{x \in U_{0}}\left|w_{J}^{\prime}(x)\right|, \quad R_{J}=\sup _{x \in U_{0}}\left|w_{J}^{\prime}(x)\right| .
$$

Lemma 2.1. Suppose $X$ and $\left\{w_{j}\right\}_{j=1}^{m}$ are defined as above.

(i) There exists a $c_{1}>1$ such that

$$
\begin{gathered}
R_{J} \leq c_{1} r_{J} \quad \text { for any } J \in \mathcal{J}, \\
c_{1}^{-1} r_{I} r_{J} \leq r_{I J} \leq c_{1} r_{I} r_{J} \quad \text { for any } I, J \in \mathcal{J} .
\end{gathered}
$$

(ii) There exist $c_{2} \geq c_{1}$ and $\delta>0$ such that for $x, y, z \in X$ with $|x-y| \leq \delta$,

$$
c_{2}^{-1}\left|w_{J}^{\prime}(z)\right| \leq \frac{\left|w_{J}(x)-w_{J}(y)\right|}{|x-y|} \leq c_{2}\left|w_{J}^{\prime}(z)\right| \quad \text { for any } J \in \mathcal{J} \text {. }
$$

(iii) There exist $c_{3} \geq c_{2}$ and $k_{0}$ such that for any $x, y \in X$,

$$
\left|w_{J}(x)-w_{J}(y)\right| \leq c_{3} r_{J}|x-y| \quad \text { for any } J \in \mathcal{J} \text { with }|J|>k_{0} .
$$

Proof. The proof of (i) and (ii) is in [FL, Lemma 2.3]. We include the proof of (ii) for completeness. For any $x \in X$, there exists $\delta_{x}>0$ such that $B\left(x, \delta_{x}\right) \subseteq U_{0}$. Since $X$ is compact, there exists $\delta>0$ (the Lebesgue number) such that for any $x, y \in X$, if $|x-y| \leq \delta$, then $x, y \in B\left(x^{\prime}, \delta_{x^{\prime}}\right)$ for some $x^{\prime} \in X$. For such $x, y \in X$, we have $w_{J}(x), w_{J}(y) \in B\left(y^{\prime}, \delta_{y^{\prime}}\right)$ for some $y^{\prime} \in X$. Then the self-similar property of $w_{J}$ implies that

$$
\left|w_{J}(x)-w_{J}(y)\right| \leq R_{J}|x-y| .
$$

On the other hand, let $u_{J}(\cdot)$ be the inverse of $w_{J}$ on $B\left(y^{\prime}, \delta_{y^{\prime}}\right) \cap w_{J}\left(B\left(x^{\prime}, \delta_{x^{\prime}}\right)\right)$, i.e.,

$$
u_{J}(x):=w_{J}^{-1}(x) \quad \text { for any } x \in B\left(y^{\prime}, \delta_{y^{\prime}}\right) \cap w_{J}\left(B\left(x^{\prime}, \delta_{x^{\prime}}\right)\right) .
$$

Then

$$
R_{J}^{-1} \leq\left|u_{J}^{\prime}(x)\right| \leq r_{J}^{-1} \quad \text { for any } x \in B\left(y^{\prime}, \delta_{y^{\prime}}\right) \cap w_{J}\left(B\left(x^{\prime}, \delta_{x^{\prime}}\right)\right) .
$$

By the self-similar property of $w_{J}(\cdot)$, we deduce that $B\left(y^{\prime}, \delta_{y^{\prime}}\right) \cap w_{J}\left(B\left(x^{\prime}, \delta_{x^{\prime}}\right)\right)$ is convex connected, hence similarly to $(2.6)$, we have

$$
\left|u_{J}\left(w_{J}(x)\right)-u_{J}\left(w_{J}(y)\right)\right| \leq r_{J}^{-1}\left|w_{J}(x)-w_{J}(y)\right| .
$$

Consequently, $r_{J}|x-y| \leq\left|w_{J}(x)-w_{J}(y)\right| \leq R_{J}|x-y|$. This together with (2.2) implies (ii).

(iii) follows directly from the choice of $\delta$ and (ii).

To make use of the local connectedness of $X$, we take $0<\varepsilon<2^{-1} c_{3}^{-1} \delta$. Then $2 c_{3} \varepsilon \leq \delta$, and hence by the assumption on $X$, we have

$$
B\left(K, c_{3} \varepsilon\right) \subseteq X .
$$

For $J \in \mathcal{J}$, let

$$
G_{J}=w_{J}(B(K, \varepsilon))
$$


Consequently, by (2.7) and (2.4), we have for any $x \in K$,

$$
B\left(w_{J}(x), c_{2}^{-1} \varepsilon r_{J}\right) \subseteq w_{J}(B(x, \varepsilon)) \subseteq B\left(w_{J}(x), c_{2} \varepsilon r_{J}\right) .
$$

It follows that

$$
\begin{aligned}
B\left(K_{J}, c_{2}^{-1} \varepsilon r_{J}\right) & =\bigcup_{x \in K} B\left(w_{J}(x), c_{2}^{-1} \varepsilon r_{J}\right) \\
& \subseteq G_{J}=\bigcup_{x \in K} w_{J}(B(x, \varepsilon)) \subseteq \bigcup_{x \in K} B\left(w_{J}(x), c_{2} \varepsilon r_{J}\right) \\
& =B\left(K_{J}, c_{2} \varepsilon r_{J}\right) .
\end{aligned}
$$

We remark that in $[\mathrm{MU}]$ and $[\mathrm{P}]$ the connectedness of $X$ was used to apply the mean value theorem so as to deduce (2.8) and (2.9); the above argument shows that the local connectedness of $X$ is sufficient. Hence we can study separation properties without assuming the connectedness of $U_{0}$ so long as we regard the relevant sets as unions of subsets whose diameters are less than $\delta$.

For $0<b<1$, we let

$$
\Lambda_{b}=\left\{J=j_{1} \ldots j_{n}: r_{j_{1} \ldots j_{n}}<b \leq r_{j_{1} \ldots j_{n-1}}\right\} .
$$

As in [L], our most crucial difference from $[\mathrm{S}]$ and $[\mathrm{P}]$ is the following inductive way of defining an index set $\Lambda(J), J \in \mathcal{J}$ : Let $k_{0}$ be as in Lemma 2.1(iii). For $J$ with $|J|=k_{0}$, we define

$$
\Lambda(J)=\left\{I \in \Lambda_{\text {diam } G_{J}}: K_{I} \cap G_{J} \neq \emptyset\right\} .
$$

Supposing $\Lambda(J)$ is defined, for any $1 \leq j \leq m$, we define $\Lambda(j J)=\mathcal{A} \cup \mathcal{B}$ where

$$
\mathcal{A}=\{j I: I \in \Lambda(J)\}, \quad \mathcal{B}=\left\{I \in \Lambda_{\text {diam } G_{j J}}: i_{1} \neq j \text { and } K_{I} \cap G_{j J} \neq \emptyset\right\} .
$$

(Note that in $[\mathrm{S}]$, the $\Lambda(J)$ is defined as $\left\{I \in \Lambda_{\text {diam } G_{J}}: K_{I} \cap G_{J} \neq \emptyset\right\}$.) It is easy to see from the construction that each $I \in \Lambda(J)$ is of type either $\mathcal{A}$ or $\mathcal{B}$, and $K_{I} \cap G_{J} \neq \emptyset$; also $K_{I}$ and $K_{J}$ are comparable in size by the following lemma.

Lemma 2.2. Suppose $\left\{w_{j}\right\}_{j=1}^{m}$ is as in Lemma 2.1. Then there exist $k_{1}$ and $c_{4}>0$ such that $c_{4}^{-1} \leq r_{J} / r_{I} \leq c_{4}$ for all $I \in \Lambda(J)$ and $J \in \mathcal{J}$ with $|J| \geq k_{1}$.

Proof. The idea is in [L, Lemma 3.1]; we modify it to fit our purpose. Let $k_{1} \geq k_{0}$ be such that

$$
\min \left\{|I|: I \in \Lambda(J) \text { and }|J| \geq k_{1}\right\}>k_{0} .
$$

For any $I \in \Lambda(J)$ and $J \in \mathcal{J}$ with $|J| \geq k_{1}$, we consider two cases:

(i) If $i_{1} \neq j_{1}$, by the construction of $\mathcal{B}$, we have $I \in \Lambda_{\text {diam } G_{J}}$. Then

$$
r_{I} \leq \operatorname{diam} G_{J} \leq r_{i_{1} \ldots i_{n-1}} \leq c_{1} r^{-1} r_{I}
$$


where $r=\min _{1 \leq j \leq m}\left\{r_{j}\right\}$. As $\varepsilon<2^{-1} c_{3}^{-1} \delta<\delta$, it follows from (2.4) that $\operatorname{diam} G_{J} \geq c_{2}^{-1} \varepsilon r_{J}$. Hence

$$
c_{2}^{-1} \varepsilon r_{J} \leq \operatorname{diam} G_{J} \leq c_{1} r^{-1} r_{I} .
$$

Also by (2.9), we have $\operatorname{diam} G_{J} \leq 2 c_{2} \varepsilon r_{J}+\left|K_{J}\right|$. Then by (2.10) and (2.5), it follows that

$$
r_{I} \leq \operatorname{diam} G_{J} \leq c_{3}(2 \varepsilon+|K|) r_{J} .
$$

Hence (2.11) and (2.12) imply that there exists $a>0$ such that

$$
a^{-1} \leq r_{J} / r_{I} \leq a
$$

(ii) If $i_{1}=j_{1}$, we write

$$
J=j_{1} \ldots j_{l} j_{l+1} \ldots j_{n}:=j_{1} \ldots j_{l} J^{\prime}, \quad I=j_{1} \ldots j_{l} i_{l+1} \ldots i_{m}:=j_{1} \ldots j_{l} I^{\prime}
$$

where $j_{l+1} \neq i_{l+1}$. Then by the construction of $\mathcal{A}$, we see inductively that $I^{\prime} \in \Lambda\left(J^{\prime}\right)$ and by (2.13), $a^{-1} \leq r_{J^{\prime}} / r_{I^{\prime}} \leq a$. Together with Lemma 2.1(i), this implies that

$$
\left(a c_{1}^{2}\right)^{-1} \leq r_{J} / r_{I} \leq a c_{1}^{2} .
$$

If we let $c_{4}=a c_{1}^{2}$, then the lemma follows from the conclusion of the two cases.

We remark that for fixed $J_{0} \in \mathcal{J}$, the construction of the set $\mathcal{A}$ implies trivially that

$$
\Lambda\left(j J_{0}\right) \supseteq\left\{j I: I \in \Lambda\left(J_{0}\right)\right\}, \quad j=1, \ldots, m .
$$

The key to proving the SOSC is to find $J_{0}$ such that equality holds (Lemma 3.4 below). In this case the set $\mathcal{B}$ is empty.

3. The proof of the main results. We need a few notations and lemmas. For any two subsets $E, F$ in $\mathbb{R}^{d}$, we define

$$
\begin{aligned}
D(E, F) & =\inf \{|x-y|: x \in E, y \in F\} ; \\
d(E, F) & =\inf \{\varepsilon: E \subseteq B(F, \varepsilon), F \subseteq B(E, \varepsilon)\} .
\end{aligned}
$$

Lemma 3.1 [FL, Lemma 2.8]. Let $w$ be conformal and invertible, let $D$ be a Borel subset in the domain of $w$, and $0<\mathcal{H}^{\alpha}(D)<\infty$. Then we have the following change of variable formula:

$$
\mathcal{H}^{\alpha}(w(D))=\int_{D}\left|w^{\prime}(x)\right|^{\alpha} d \mathcal{H}^{\alpha}(x) .
$$

Lemma 3.2. Let $\left\{w_{j}\right\}_{j=1}^{m}$ be as in Theorem 1.1. Suppose $0<\mathcal{H}^{\alpha}(K)$ $<\infty$. Then

$$
\mathcal{H}^{\alpha}\left(K_{I} \cap K_{J}\right)=0 \quad \text { for any incomparable } I, J \in \mathcal{J} .
$$


Proof. Since $T_{\alpha}$ has spectral radius 1, by [FL, Theorem 1.1], there exists $0<h \in C(K)$ such that $h(x)=\sum_{j=1}^{m}\left|w_{j}^{\prime}(x)\right|^{\alpha} h\left(w_{j} x\right)$. Then

$$
\begin{aligned}
\sum_{j=1}^{m} \int_{K_{j}} h(x) d \mathcal{H}^{\alpha}(x) & \geq \int_{\bigcup_{j=1}^{m} K_{j}} h(x) d \mathcal{H}^{\alpha}(x)=\int_{K} h(x) d \mathcal{H}^{\alpha}(x) \\
= & \int_{K} \sum_{j=1}^{m}\left|w_{j}^{\prime}(x)\right|^{\alpha} h\left(w_{j} x\right) d \mathcal{H}^{\alpha}(x)=\sum_{j=1}^{m} \int_{K_{j}} h(x) d \mathcal{H}^{\alpha}(x) .
\end{aligned}
$$

(The last equality follows from Lemma 3.1.) This implies that $\mathcal{H}^{\alpha}\left(K_{i} \cap K_{j}\right)$ $=0$ for any $i \neq j$. It follows immediately that $\mathcal{H}^{\alpha}\left(K_{I} \cap K_{J}\right)=0$ for any incomparable $I, J \in \mathcal{J}$.

Lemma 3.3. Let $\left\{w_{j}\right\}_{j=1}^{m}$ be as in Lemma 3.2. Then there exists $\delta_{0}>0$ such that for any $L \in \mathcal{J}$,

$$
\left\|w_{I}(\cdot)-w_{J}(\cdot)\right\|_{C(K)} \geq \delta_{0} r_{L} \quad \text { for any } I, J \in \Lambda(L) \text { with } I \neq J .
$$

Proof. Since $0<\mathcal{H}^{\alpha}(K)<\infty$, there exists an open set $U$ such that $K \subseteq U \subset X$ and

$$
0<\mathcal{H}^{\alpha}(U) \leq \mathcal{H}^{\alpha}(K)+1<\infty .
$$

Let $c_{1}$ and $\delta$ be as in Lemma 2.1 and let $0<\eta<2^{-1} c_{1}^{-\alpha}$. There exists an open covering $\left\{V_{i}\right\}_{i=1}^{n}$ of $K$ such that

$$
\begin{gathered}
K \subseteq V:=\bigcup_{i=1}^{n} V_{i} \subseteq U, \quad \delta^{\prime}:=D\left(K, V^{\mathrm{c}}\right)<\delta, \\
0<\mathcal{H}^{\alpha}(K) \leq \mathcal{H}^{\alpha}(V) \leq \sum_{i=1}^{n}\left|V_{i}\right|^{\alpha}<(1+\eta) \mathcal{H}^{\alpha}(K) .
\end{gathered}
$$

For any $I, J \in \Lambda(L)$, assume without loss of generality that $\mathcal{H}^{\alpha}\left(K_{I}\right) \leq$ $\mathcal{H}^{\alpha}\left(K_{J}\right)$. Then for any given $\varepsilon$ satisfying $c_{1}^{\alpha} \eta<\varepsilon<1$, we have

$$
\varepsilon \mathcal{H}^{\alpha}\left(K_{I}\right)<\mathcal{H}^{\alpha}\left(K_{J}\right)
$$

We claim that $d\left(K_{I}, K_{J}\right) \geq \delta^{\prime} r_{I}$. Otherwise, by (3.1) and Lemma 2.1(ii), we have $D\left(K_{I}, w_{I}\left(V^{\mathrm{c}}\right)\right) \geq \delta^{\prime} r_{I}$, and then $K_{J} \subseteq w_{I}(V)$. Hence by (3.3) and Lemma 3.2, we have

$$
(1+\varepsilon) \mathcal{H}^{\alpha}\left(K_{I}\right)<\mathcal{H}^{\alpha}\left(K_{I}\right)+\mathcal{H}^{\alpha}\left(K_{J}\right)=\mathcal{H}^{\alpha}\left(K_{I} \cup K_{J}\right) \leq \mathcal{H}^{\alpha}\left(w_{I}(V)\right) .
$$

This together with (3.2) implies that

$$
\begin{aligned}
\varepsilon_{I}^{\alpha} \mathcal{H}^{\alpha}(K) & \leq \varepsilon \mathcal{H}^{\alpha}\left(K_{I}\right)<\mathcal{H}^{\alpha}\left(w_{I}(V \backslash K)\right) \leq\left(c_{1} r_{I}\right)^{\alpha} \mathcal{H}^{\alpha}(V \backslash K) \\
& <\left(c_{1} r_{I}\right)^{\alpha} \eta \mathcal{H}^{\alpha}(K) .
\end{aligned}
$$

(The first and third inequalities follow from Lemmas 3.1 and 2.1(i).) Then $\varepsilon<c_{1}^{\alpha} \eta$, which contradicts the choice of $\varepsilon$. The claim is proved, and the lemma follows. 
Lemma 3.4. Let $\left\{w_{j}\right\}_{j=1}^{m}$ be as in Lemma 3.2. Then $\gamma:=\sup _{|L| \geq k_{1}} \sharp \Lambda(L)$ $<\infty$. If $J_{0} \in \mathcal{J}$ is such that $\left|J_{0}\right| \geq k_{1}$ and $\sharp \Lambda\left(J_{0}\right)=\gamma$, then

$$
\Lambda\left(I J_{0}\right)=\left\{I J: J \in \Lambda\left(J_{0}\right)\right\} \quad \text { for all } I \in \mathcal{J} .
$$

Proof. Let $c_{3}, c_{4}$ and $\delta_{0}$ be the constants given in Lemmas 2.1, 2.2 and 3.3 respectively. Let $\delta^{\prime}=\left(3 c_{3} c_{4}\right)^{-1} \delta_{0}$. We can find a finite set $Z \subset K$ whose $\delta^{\prime}$-neighborhood contains $K$. For any $L \in \mathcal{J}$ with $|L| \geq k_{1}$ and for all different $I, J \in \Lambda(L)$, by Lemma 3.3, there exists $x \in K$ such that $\left|w_{I}(x)-w_{J}(x)\right| \geq \delta_{0} r_{L}$. For that $x$ there exists $z \in Z$ such that $|x-z|<\delta^{\prime}$; then by (2.5) and the choice of $k_{1}$ (see the proof of Lemma 2.2), we have

$$
\left|w_{I}(x)-w_{I}(z)\right| \leq \frac{1}{3} \delta_{0} r_{L} \quad \text { and } \quad\left|w_{J}(x)-w_{J}(z)\right| \leq \frac{1}{3} \delta_{0} r_{L} .
$$

It follows that for any different $I, J \in \Lambda(L)$, there exists some $z \in Z$ such that

$$
\left|w_{I}(z)-w_{J}(z)\right| \geq \frac{1}{3} \delta_{0} r_{L}
$$

For each $z \in Z$, set

$$
P_{z}(L)=\{I \in \Lambda(L): \exists J \in \Lambda(L) \text { such that (3.5) holds }\} .
$$

Hence (3.5) implies that

$$
\Lambda(L)=\bigcup_{z \in Z} P_{z}(L)
$$

To prove $\sup _{|L| \geq k_{1}} \sharp \Lambda(L)<\infty$, we observe that for each $z \in Z$, the sets

$$
\left\{B\left(w_{I}(z), \frac{1}{6} \delta_{0} r_{L}\right): I \in P_{z}(L)\right\}
$$

are disjoint by $(3.5)$ and are contained in $B\left(G_{L}\right.$, diam $\left.K_{I}+\frac{1}{6} \delta_{0} r_{L}\right)$ by the definition of $\Lambda(L)$. By Lemma 2.1(iii) and Lemma 2.2, there exist $c>0$ (independent of $L)$ and $x \in K$ such that $B\left(G_{L}\right.$, diam $\left.K_{I}+\frac{1}{6} \delta_{0} r_{L}\right) \subseteq B\left(x, c r_{L}\right)$. By a simple volume argument, we deduce that there exists an $\ell$ (independent of $L$ ) such that $\max _{z \in Z} \sharp P_{z}(L) \leq \ell$. Then

$$
\sharp \Lambda(L) \leq \sharp Z \cdot \max _{z \in Z} \sharp P_{z}(L) \leq \ell \cdot \sharp Z .
$$

We conclude that $\gamma=\sup _{|L| \geq k_{1}} \sharp \Lambda(L)<\infty$. Hence there exists $J_{0}$ such that $\left|J_{0}\right| \geq k_{1}$ and $\sharp \Lambda\left(J_{0}\right)=\gamma$.

To prove (3.4), we have remarked after the definition of $\Lambda(J)$ that

$$
\Lambda\left(j J_{0}\right) \supseteq\left\{j I: I \in \Lambda\left(J_{0}\right)\right\}, \quad j=1, \ldots, m .
$$

On the other hand, the choice of $J_{0}$ implies that $\sharp\left\{I J: J \in \Lambda\left(J_{0}\right)\right\}=\gamma$. Thus the definition of $\gamma$ implies that $\sharp \Lambda\left(I J_{0}\right)=\gamma$ also and (3.4) follows.

Proof of Theorem 1.1. It is obvious that (ii) implies (i). That (i) implies (iii) is shown in $[\mathrm{MU}]$ and $[\mathrm{FL}]$. We have to prove (iii) $\Rightarrow$ (ii). The proof needs only a small modification of $[\mathrm{S}]$ and is the same as in $[\mathrm{L}]$; we include it here 
for completeness. Let $J_{0} \in \mathcal{J}$ be as in Lemma 3.4. For any fixed $1 \leq l \leq m$ and $J=j_{1} \ldots j_{n} \in \mathcal{J}$ with $j_{1} \neq l$, we consider the family

$$
\mathcal{K}_{l}=\left\{K_{L}: L \in \Lambda_{\operatorname{diam} G_{J J}} \text { with } l_{1}=l\right\}
$$

where $l_{1}$ is the first element of the multiple index $L$. Then $\mathcal{K}_{l}$ is a cover of $K_{l}$. Since $j_{1} \neq l_{1},(3.4)$ implies that $L \notin \Lambda\left(J J_{0}\right)$. Then by the construction of $\mathcal{B}, K_{L} \cap G_{J J_{0}}=\emptyset$. Hence by $(2.9)$, we have $D\left(K_{L}, K_{J J_{0}}\right) \geq c_{2}^{-1} \varepsilon r_{J J_{0}}$, which implies

$$
D\left(K_{l}, K_{J J_{0}}\right) \geq c_{2}^{-1} \varepsilon r_{J J_{0}} \quad \text { for } l \neq j_{1} .
$$

Now we let $G_{J}^{*}=w_{J}\left(B\left(K, 2^{-1} c_{2}^{-2} \varepsilon\right)\right)$ and

$$
U=\bigcup_{J \in \mathcal{J}} G_{J J_{0}}^{*}
$$

We claim that the $U$ satisfies the condition of the SOSC. Indeed, $U$ is a bounded open set, $U \cap K \neq \emptyset$ and

$$
w_{j}(U)=\bigcup_{J \in \mathcal{J}} w_{j}\left(G_{J J_{0}}^{*}\right)=\bigcup_{J \in \mathcal{J}} G_{j J J_{0}}^{*} \subseteq U .
$$

Now we prove that

$$
w_{i}(U) \cap w_{j}(U)=\emptyset \quad \text { for } i \neq j .
$$

For otherwise, there are $I, J$ such that $G_{i I J_{0}}^{*} \cap G_{j J J_{0}}^{*} \neq \emptyset$. We assume $r_{i I J_{0}} \geq$ $r_{j J J_{0}}$. Let $y$ be in the intersection; then there exist $y_{1} \in K_{i I J_{0}}$ and $y_{2} \in K_{j J J_{0}}$ such that

$$
\begin{aligned}
& d\left(y, y_{1}\right)<c_{2} \cdot \frac{1}{2 c_{2}^{2}} \varepsilon \cdot r_{i I J_{0}} \leq \frac{c_{2}^{-1} \varepsilon}{2} r_{i I J_{0}} \\
& d\left(y, y_{2}\right)<c_{2} \cdot \frac{1}{2 c_{2}^{2}} \varepsilon \cdot r_{j J J_{0}} \leq \frac{c_{2}^{-1} \varepsilon}{2} r_{i I J_{0}} .
\end{aligned}
$$

Then $d\left(y_{1}, y_{2}\right)<c_{2}^{-1} \varepsilon r_{i I J_{0}}$. Hence

$$
D\left(K_{i I J_{0}}, K_{j}\right)<c_{2}^{-1} \varepsilon r_{i I J_{0}}
$$

which contradicts (3.6). This completes the proof.

Lemma 3.5 [FL, Theorem 2.9]. Let $\left\{w_{j}\right\}_{j=1}^{m}$ be as in Theorem 1.1 and satisfy the $O S C$. Let $\nu=\left.\mathcal{H}^{\alpha}\right|_{K}$. Then $\nu$ is an invariant measure for $T_{\alpha}$, i.e., $T_{\alpha}^{*} \nu=\nu$.

Proof of Theorem 1.2. By assumption and Theorem 1.1, we have $0<$ $\mathcal{H}^{\alpha}(K)<\infty$. We recall the proof of Theorem 1.1 and let $U$ be as constructed there. To prove $\operatorname{dim}_{H}(K \backslash U)<\alpha$, let $\mu=\mathcal{H}^{\alpha}(K)^{-1} \mathcal{H}^{\alpha}$. Then by Lemma 3.5, $\mu$ is an invariant probability measure of $T_{\alpha}$, i.e.,

$$
\mu=\sum_{j=1}^{m}\left(\left|w_{j}^{\prime}(x)\right|^{\alpha} \mu\right) \circ w_{j}^{-1} \text {. }
$$


Let $k:=\left|J_{0}\right|$. Then by Lemmas 2.1 and 3.1, we have

$$
\mu\left(K_{J_{0}}\right) \geq c_{1}^{-\alpha} r_{J_{0}}^{\alpha}
$$

for any $L \in \mathcal{J}$,

$$
\begin{aligned}
\mu\left(K_{L J_{0}}\right) & =\mathcal{H}^{\alpha}(K)^{-1} \int_{K}\left|w_{L}^{\prime}\left(w_{J_{0}} x\right)\right|^{\alpha}\left|w_{J_{0}}^{\prime}(x)\right|^{\alpha} d \mathcal{H}^{\alpha}(x) \\
& \geq r_{L}^{\alpha} r_{J_{0}}^{\alpha} \geq c_{1}^{-\alpha} r_{J_{0}}^{\alpha} \mu\left(K_{L}\right) .
\end{aligned}
$$

For any integer $n$, let

$$
U_{n}=\bigcup_{\ell=0}^{n-1} \bigcup_{|J|=k \ell} G_{J J_{0}}^{*}
$$

Then $U_{n} \subseteq U$. Let

$$
\begin{aligned}
\mathcal{J}(n) & =\left\{j_{1} \ldots j_{k n}: 1 \leq j_{i} \leq m\right\}, \\
\mathcal{L}_{n} & =\left\{L=l_{1} \ldots l_{k n} \in \mathcal{J}(n): l_{k \ell+1} \ldots l_{k \ell+k} \neq J_{0} \forall 0 \leq \ell<n\right\} .
\end{aligned}
$$

For any $J$ with $0 \leq|J|=k \ell<k n$, we deduce from $K=\bigcup_{j=1}^{m} w_{j}(K)$ that

$$
K_{J J_{0}}=w_{J J_{0}}(K)=\bigcup_{\left|J^{\prime}\right|=k(n-1)-|J|} K_{J J_{0} J^{\prime}}
$$

Then

$$
\begin{aligned}
K \backslash U & \subseteq K \backslash U_{n}=K \backslash \bigcup_{\ell=0}^{n-1} \bigcup_{|J|=k \ell} G_{J J_{0}}^{*} \subseteq K \backslash \bigcup_{\ell=0}^{n-1} \bigcup_{|J|=k \ell} K_{J J_{0}} \\
& =\bigcap_{\ell=0}^{n-1} \bigcap_{|J|=k \ell} \bigcap_{\left|J^{\prime}\right|=k(n-1)-|J|} K_{J J_{0} J^{\prime}}^{\mathrm{c}} \subseteq \bigcup_{L \in \mathcal{L}_{n}} K_{L} .
\end{aligned}
$$

We need to estimate the value of $\mathcal{H}^{\alpha}\left(\bigcup_{L \in \mathcal{L}_{n}} K_{L}\right)$. For this we will prove inductively that

$$
\sum_{L \in \mathcal{L}_{n}} \mu\left(K_{L}\right) \leq\left(1-c_{1}^{-\alpha} r_{J_{0}}^{\alpha}\right)^{n} \quad \text { for any } n .
$$

Indeed, by Lemma 3.2 , we have $\mu\left(K_{I} \cap K_{J}\right)=0$ for any $I, J \in \mathcal{L}_{n}$ with $I \neq J$. This together with (3.8) implies that

$$
\sum_{L \in \mathcal{L}_{1}} \mu\left(K_{L}\right)=\mu\left(\bigcup_{\substack{L \neq J_{0} \\|L|=k}} K_{L}\right)=1-\mu\left(K_{J_{0}}\right) \leq 1-c_{1}^{-\alpha} r_{J_{0}}^{\alpha} .
$$

Assume that

$$
\sum_{L \in \mathcal{L}_{n}} \mu\left(K_{L}\right) \leq\left(1-c_{1}^{-\alpha} r_{J_{0}}^{\alpha}\right)^{n}
$$


Since $\sum_{|J|=k} \mu\left(K_{L J}\right)=\mu\left(K_{L}\right)$, we have

$$
\begin{aligned}
\sum_{L \in \mathcal{L}_{n+1}} \mu\left(K_{L}\right) & =\sum_{L \in \mathcal{L}_{n}} \sum_{|J|=k} \mu\left(K_{L J}\right)-\sum_{L \in \mathcal{L}_{n}} \mu\left(K_{L J_{0}}\right) \\
& =\sum_{L \in \mathcal{L}_{n}} \mu\left(K_{L}\right)-\sum_{L \in \mathcal{L}_{n}} \mu\left(K_{L J_{0}}\right) \\
& \leq \sum_{L \in \mathcal{L}_{n}} \mu\left(K_{L}\right)-c_{1}^{-\alpha} r_{J_{0}}^{\alpha} \sum_{L \in \mathcal{L}_{n}} \mu\left(K_{L}\right) \quad \text { by }(3.9) \\
& =\left(1-c_{1}^{-\alpha} r_{J_{0}}^{\alpha}\right) \sum_{L \in \mathcal{L}_{n}} \mu\left(K_{L}\right) \leq\left(1-c_{1}^{-\alpha} r_{J_{0}}^{\alpha}\right)\left(1-c_{1}^{-\alpha} r_{J_{0}}^{\alpha}\right)^{n} \\
& =\left(1-c_{1}^{-\alpha} r_{J_{0}}^{\alpha}\right)^{n+1} .
\end{aligned}
$$

Let $\delta_{n}:=\max \left\{\operatorname{diam} K_{L}: L \in \mathcal{L}_{n}\right\}$ and $r=\min _{1 \leq j \leq m}\left\{r_{j}\right\}$. Take

$$
\beta:=\alpha-\frac{\log \left(1-c_{1}^{-\alpha} r_{J_{0}}^{\alpha}\right)}{k \log r} .
$$

Then $\beta<\alpha$. Set $c_{4}=\left(c_{3} \operatorname{diam} K\right)^{\beta}$. Then for large $n$, we have

$$
\begin{aligned}
& \mathcal{H}_{\delta_{n}}^{\beta}(K \backslash U) \leq \mathcal{H}_{\delta_{n}}^{\beta}\left(\bigcup_{L \in \mathcal{L}_{n}} K_{L}\right) \leq \sum_{L \in \mathcal{L}_{n}}\left(\operatorname{diam} K_{L}\right)^{\beta} \quad \text { by }(3.11) \\
& \quad \leq \sum_{L \in \mathcal{L}_{n}}\left(c_{3} r_{L} \operatorname{diam} K\right)^{\beta}=c_{4} \sum_{L \in \mathcal{L}_{n}} r_{L}^{\beta}=c_{4} \sum_{L \in \mathcal{L}_{n}} r_{L}^{\beta-\alpha} r_{L}^{\alpha} \quad \text { by } \quad(2.5) \\
& \leq c_{4}\left(r^{n k(\beta-\alpha)} \sum_{L \in \mathcal{L}_{n}} \mu\left(K_{L}\right)\right) \leq c_{4}\left(r^{k(\beta-\alpha)}\left(1-c_{1}^{-\alpha} r_{J_{0}}^{\alpha}\right)\right)^{n} \leq c_{4} \quad \text { by }(3.12)
\end{aligned}
$$

Since $\lim _{n \rightarrow \infty} \delta_{n}=0$, we obtain $\mathcal{H}^{\beta}(K \backslash U) \leq c_{4}<\infty$, hence $\operatorname{dim}_{\mathrm{H}}(K \backslash U)$ $\leq \beta$.

Corollary 3.6. Let $\left\{w_{j}\right\}_{j=1}^{m}$ be as in Theorem 1.2. Then

$$
\operatorname{dim}_{\mathrm{H}}\left(w_{i}(K) \cap w_{j}(K)\right)<\alpha \quad \text { for } i \neq j
$$

Proof. Let $J_{0}$ and $\beta$ be as in the proof of Theorem 1.2. Using (3.12), we can show similarly to [LX, Theorem 1.6] that $\operatorname{dim}_{\mathrm{H}}\left(w_{i}(K) \cap w_{j}(K)\right)$ $\leq \beta<\alpha$.

TheOREM 3.7. Let $\left\{w_{j}\right\}_{j=1}^{m}$ be as in Theorem 1.2. If there is a basic open set $U$ such that $U \backslash \bigcup_{j=1}^{m} w_{j}(\bar{U}) \neq \emptyset$, then $\operatorname{dim}_{\mathrm{H}} K<d$.

Proof. Suppose that $\operatorname{dim}_{\mathrm{H}} K=d$. Since $\left\{w_{j}\right\}_{j=1}^{m}$ satisfies the OSC, we know from [FL, Theorem 2.7] that $T_{d}$ has spectral radius 1 , i.e., $\alpha=d$. Since $U \backslash \bigcup_{j=1}^{m} w_{j}(\bar{U})$ is an open subset of $\mathbb{R}^{d}$, the proof will be finished if we can 
show that $\mathcal{H}^{d}\left(U \backslash \bigcup_{j=1}^{m} w_{j}(\bar{U})\right)=0$. For this, let

$$
V=U \backslash \bigcup_{j=1}^{m} w_{j}(\bar{U})
$$

We claim that

$$
w_{I}(V) \cap w_{J}(V)=\emptyset \quad \forall I, J \in \mathcal{J}, I \neq J .
$$

In fact, for $I, J$ comparable, we have $J=I I_{0}$. Since $U$ is a basic open set, we have

$$
w_{i}(U) \subset U \quad \text { and } \quad w_{i}(U) \cap w_{j}(U)=\emptyset, \quad \forall i \neq j .
$$

Therefore $w_{I_{0}}(V) \subset \bigcup_{j=1}^{m} w_{j}(U)$, and thus $w_{I_{0}}(V) \cap V=\emptyset$. Hence

$$
w_{I}(V) \cap w_{J}(V) \subseteq w_{I}\left(V \cap w_{I_{0}}(V)\right)=\emptyset .
$$

If $I, J$ are incomparable, let $I=i_{1} \ldots i_{p}, J=j_{1} \ldots j_{q}$ and $r=\min \left\{k: i_{k} \neq j_{k}\right\}$. Define $I_{0}=i_{1} \ldots i_{r-1}$. By (3.13) and (3.15), we have

$$
w_{I}(V) \cap w_{J}(V) \subseteq w_{I_{0}}\left(w_{i_{r}}(U) \cap w_{j_{r}}(U)\right)=\emptyset .
$$

This completes the proof of the claim.

By (3.14) and Lemma 3.1, we have

$$
\begin{aligned}
\sum_{n=1}^{\infty} \int_{V|J|=n} \sum_{V}\left|w_{J}^{\prime}(x)\right|^{d} d \mathcal{H}^{d}(x) & =\sum_{n=1}^{\infty} \sum_{|J|=n} \mathcal{H}^{d}\left(w_{J}(V)\right) \\
& =\mathcal{H}^{d}\left(\bigcup_{J \in \mathcal{J}} w_{J}(V)\right) \leq \mathcal{H}^{d}(U)<\infty
\end{aligned}
$$

On the other hand, for any fixed $y_{0} \in K$ and any $x \in X$, by Lemma 2.1(i),

$$
c_{1}^{-d}\left|w_{J}^{\prime}\left(y_{0}\right)\right|^{d} \leq\left|w_{J}^{\prime}(x)\right|^{d} \text {. }
$$

Hence

$$
c_{1}^{-d} \sum_{|J|=n}\left|w_{J}^{\prime}\left(y_{0}\right)\right|^{d} \leq \sum_{|J|=n}\left|w_{J}^{\prime}(x)\right|^{d}, \quad x \in X .
$$

Since $\alpha=d$, it follows from [FL, Theorem 1.1] that

$$
\lim _{n} \sum_{|J|=n}\left|w_{J}^{\prime}(\cdot)\right|^{d}=h(\cdot) \quad \text { uniformly on } K
$$

where $0<h \in C(K)$ is the 1-eigenfunction of the Ruelle operator $T_{d}$. Then

$$
\begin{aligned}
c_{1}^{-d} h\left(y_{0}\right) \mathcal{H}^{d}(V) & =c_{1}^{-d} \cdot \lim _{n} \int_{V|J|=n}\left|w_{J}^{\prime}\left(y_{0}\right)\right|^{d} d \mathcal{H}^{d}(x) \\
& \leq \liminf _{n} \int_{V|J|=n}\left|w_{J}^{\prime}(x)\right|^{d} d \mathcal{H}^{d}(x) .
\end{aligned}
$$

By (3.16), the right side is 0 , hence $\mathcal{H}^{d}(V)=\mathcal{H}^{d}\left(U \backslash \bigcup_{j=1}^{m} w_{j}(\bar{U})\right)=0$. 
Corollary 3.8. Let $\left\{w_{j}\right\}_{j=1}^{m}$ be as in Theorem 1.1. If $\alpha=d$ and $\mathcal{H}^{d}(K)>0$, then $K^{\circ} \neq \emptyset$ and $\operatorname{dim}_{\mathrm{H}} \partial K<d$.

Proof. Let $U$ be the basic open set constructed in the proof of Theorem 1.1. By assumption and Theorem 3.7, we have $\mathcal{H}^{d}\left(U \backslash \bigcup_{j=1}^{m} w_{j}(\bar{U})\right)=0$. Then

$$
\bar{U}=\bigcup_{j=1}^{m} w_{j}(\bar{U}) .
$$

By the uniqueness of the invariant set $K$, we have $K=\bar{U}$, and then $K^{\circ} \supseteq$ $U \neq \emptyset$. In view of the proof of Theorem 1.2, we have

$$
\operatorname{dim}_{\mathrm{H}} \partial K \leq \operatorname{dim}_{\mathrm{H}}(K \backslash U)<d
$$

Acknowledgements. I would like to express my deepest gratitude to Professor Ka-Sing Lau for numerous valuable discussions and for going through the manuscript. Thanks are also due to Dr. H. Rao for valuable discussions.

\section{References}

[BG] C. Bandt and S. Graf, Self-similar sets. VII. A characterization of self-similar fractals with positive Hausdorff measure, Proc. Amer. Math. Soc. 114 (1992), 995-1001.

[FL] A. H. Fan and K. S. Lau, Iterated function system and Ruelle operator, J. Math. Anal. Appl. 231 (1999), 319-344.

$[\mathrm{H}] \quad$ J. Hutchinson, Fractals and self-similarity, Indiana Univ. Math. J. 30 (1981), 713-747.

[L] K. S. Lau, H. Rao and Y. L. Ye, Corrigendum: "Iterated function system and Ruelle operator", J. Math. Anal. Appl. 262 (2001), 446-451.

[LX] K. S. Lau and Y. Xu, On the boundary of attractors with non-void interior, Proc. Amer. Math. Soc. 128 (2000), 1761-1768.

[MU] R. D. Mauldin and M. Urbański, Dimensions and measures in infinite iterated function systems, Proc. London Math. Soc. 73 (1996), 106-154.

[P] Y. Peres, M. Rams, K. Simon and B. Solomyak, Equivalence of positive Hausdorff measure and the open set condition for self-conformal sets, Proc. Amer. Math. Soc. 129 (2001), 2689-2699.

[S] A. Schief, Separation properties for self-similar sets, ibid. 122 (1994), 111-115.

Department of Mathematics

The Chinese University of Hong Kong

Shatin, Hong Kong

E-mail: ylye@math.cuhk.edu.hk
Present address:

Department of Mathematics South China Normal University Guangzhou 510631, P.R. China E-mail: ylye@scnu.edu.cn 